ARTICLE

\title{
Stable single atomic silver wires assembling into a circuitry-connectable nanoarray
}

\author{
Yaxin Chen (1) 1,11, Daiming Tang (1) 2,11, Zhiwei Huang 1,3,11, Xi Liu 4,5,11, Jun Chen², Takashi Sekiguchi ${ }^{2,6}$,

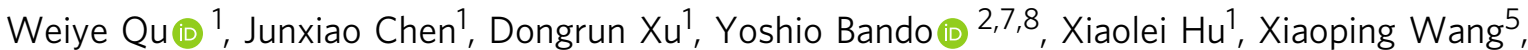 \\ Dmitri Golberg ${ }^{2,9}$ \& Xingfu Tang (1) ${ }^{1,10 凶}$
}

\begin{abstract}
Atomic metal wires have great promise for practical applications in devices due to their unique electronic properties. Unfortunately, such atomic wires are extremely unstable. Here we fabricate stable atomic silver wires (ASWs) with appreciably unoccupied states inside the parallel tunnels of $\alpha-\mathrm{MnO}_{2}$ nanorods. These unoccupied $\mathrm{Ag} 4 d$ orbitals strengthen the $\mathrm{Ag}-\mathrm{Ag}$ bonds, greatly enhancing the stability of ASWs while the presence of delocalized $5 s$ electrons makes the ASWs conducting. These stable ASWs form a coherently oriented threedimensional wire array of over $10 \mathrm{~nm}$ in width and up to $1 \mu \mathrm{m}$ in length allowing us to connect it to nano-electrodes. Current-voltage characteristics of ASWs show a temperaturedependent insulator-to-metal transition, suggesting that the atomic wires could be used as thermal electrical devices.
\end{abstract}

\footnotetext{
${ }^{1}$ Department of Environmental Science and Engineering, Fudan University, Shanghai, China. ${ }^{2}$ World Premier International Center for Materials Nanoarchitectonics, National Institute for Materials Science, Namiki 1-1, Tsukuba, Ibaraki, Japan. ${ }^{3}$ Department of Environmental Science and Engineering, College of Chemical Engineering, Huaqiao University, Xiamen, Fujian, China. ${ }^{4}$ School of Chemistry and Chemical, In-situ Center for Physical Science, Shanghai Jiao Tong University, Shanghai, China. ${ }^{5}$ Syncat@Beijing, SynfuelsChina Co. Ltd, Beijing, China. ${ }^{6}$ Faculty of Pure and Applied Science, University of Tsukuba, Tsukuba, Japan. ${ }^{7}$ Australian Institute for Innovative Materials, University of Wollongong, Wollongong, NSW, Australia. ${ }^{8}$ Institute of Molecular Plus, Tianjin University, Tianjin, China. ${ }^{9}$ Centre for Materials Science and School of Chemistry and Physics, Queensland University of Technology (QUT), Brisbane QLD, Australia. ${ }^{10}$ Shanghai Institute of Pollution Control and Ecological Security, Shanghai, China. ${ }^{11}$ These authors contributed equally: Yaxin Chen, Daiming Tang, Zhiwei Huang, Xi Liu. ${ }^{凶}$ email: tangxf@fudan.edu.cn
} 
O ne-dimensional (1D) atomic metal wires are promising materials as building blocks in electronic nanodevices because their electronic characteristics are distinct from those of their bulk counterparts ${ }^{1-5}$. For practical applications of atomically thin metal wires in nanoelectronics, high stability in air at room temperature and a suitable length for convenient connection into nanocircuitry are key requirements. However, the high degree of coordinative unsaturation makes the formation of long atomic metal wires extremely challenging.

Over the past two decades, the creation of long atomic metal wires through (self-)assembly techniques have been reported only in a few cases. Atomic silver wires (ASWs) of up to $100 \mathrm{~nm}$ in length were synthesized on $\mathrm{Pt}(997)$ surfaces under ultrahigh vacuum conditions ${ }^{6}$. Infinite linear atomic metal wires were fabricated in the tunnels of an air-sensitive inorganic subnitride ${ }^{7}$. Using a scanning tunneling microscope, 1D atomic wires of gold, silver, or manganese with controllable lengths were constructed on surfaces by self-assembling ${ }^{3,5,8}$. However, these long atomic wires are unstable, and thus difficult to use in practical applications $^{7-9}$. Owing to the atomically thin wire width, assembling many long atomic wires as a coherently oriented array is highly desirable for constructing miniaturized device architectures.

Herein, we create stable ASWs of up to $1 \mu \mathrm{m}$ in length by a self-assembly technique, leading to a coherently oriented threedimensional (3D) array inside the tunnels of an insulating $\alpha$ $\mathrm{MnO}_{2}$ nanorod, the stability of which derives from the scaffolding function of $\alpha-\mathrm{MnO}_{2}$ and the strengthened $\mathrm{Ag}-\mathrm{Ag}$ bonds due to the appreciably unoccupied states of the $\mathrm{Ag} 4 d$ orbitals. The stable atomic wire array with suitable $3 \mathrm{D}$ sizes can be conveniently connected with nanoelectrodes for conductance measurements. Current-voltage (I-V) data demonstrate a temperature-controlled insulator-to-metal transition, making ASWs attractive for application as thermal electrical devices.

\section{Results}

Geometric structures of the atomic wire array. ASWs were synthesized by a thermal diffusion method starting from Ag nanoparticles (NPs) supported on surfaces of the $\alpha-\mathrm{MnO}_{2}$ nanorods (see the "Methods" section for more details). The accurate geometrical structures of ASWs were investigated by synchrotron X-ray diffraction (SXRD) patterns, extended X-ray absorption fine structure (EXAFS) spectroscopy and transmission electron microscopy (TEM) imaging. TEM data shows that the incorporation of $\mathrm{Ag}$ atoms hardly changed the morphology of a$\mathrm{MnO}_{2}$ nanorods (Supplementary Figs. 1 and 2). We further conducted a Rietveld refinement of room-temperature SXRD of ASWs inside the $\alpha-\mathrm{MnO}_{2}$ tunnels together with the pristine $\alpha$ $\mathrm{MnO}_{2}$ (Supplementary Fig. 3) ${ }^{10}$. The resulting lattice information and structural parameters were summarized in Supplementary Tables 1 and 2. These data indicate that the Ag atoms of ASWs are located at the Wychoff $2 \mathrm{a}$ sites inside the $\alpha-\mathrm{MnO}_{2}$ tunnels ${ }^{11}$, and thus the closest $\mathrm{Ag}-\mathrm{Ag}$ and $\mathrm{Ag}-\mathrm{O}$ distances are determined to be 2.87 and $2.48 \AA$, respectively.

The local structure of Ag atoms in ASWs was further explored by EXAFS spectra at the Ag $K$-edge using both Fourier and wavelet transforms (Fig. 1). The wavelet transform plots show that the maxima of the wavelet transforms of $\mathrm{Ag}$ foil and $\mathrm{Ag}_{2} \mathrm{O}$ are $\sim 8$ and $\sim 7 \AA^{-1}$, which correspond to $\mathrm{Ag}-\mathrm{Ag}$ and $\mathrm{Ag}-\mathrm{O}$ scattering paths, respectively. Compared with the two references, ASWs have $k$ maxima at $\sim 5, \sim 6$, and $\sim 7 \AA^{-1}$, which should correspond to the first-shell $\mathrm{Ag}-\mathrm{O}$, metallic $\mathrm{Ag}-\mathrm{Ag}$, and secondshell $\mathrm{Ag}-\mathrm{O} / \mathrm{Mn}$ scattering paths, respectively. To verify this inference, we conducted EXAFS fitting to unveil the quantitative coordination configuration of $\mathrm{Ag}$ atoms. The fitting results show that in ASWs the interatomic distances in the two nearest
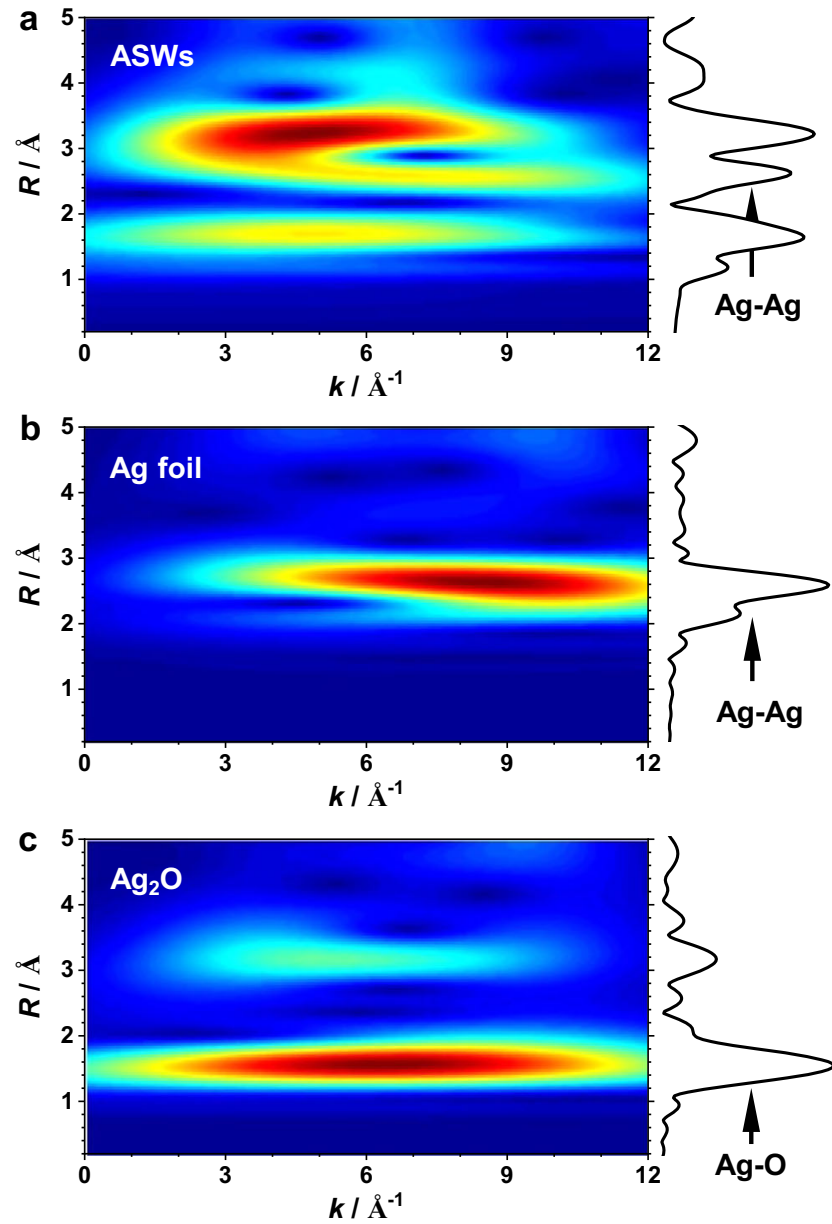

Fig. 1 Local structures of $\mathbf{A g}$ atoms in ASWs. Wavelet transform plots and $k^{3}$-weighted EXAFS spectra in $R$-space of $\mathrm{Ag}(\mathbf{a}), \operatorname{ASWs}(\mathbf{b})$, and $\mathrm{Ag}_{2} \mathrm{O}$ (c) Color scale: dark blue to red refers to low intensity to high intensity.

neighbor shells are attributed to Ag-O ( 2.48 $\mathrm{A})$ and $\mathrm{Ag}-\mathrm{Ag}$ $(\sim 2.87 \AA)$ bonds with coordination numbers (CNs) of 4 and 2 , respectively (Supplementary Fig. 4 and Table 3 ). These findings agree with the data of the SXRD refinements above. The average $\mathrm{Ag}-\mathrm{Ag}$ bond length is close to that of $2.89 \AA$ in bulk $\mathrm{Ag}$ (Supplementary Fig. 5 and Table 3$)^{12}$, implying that there is a strong Ag-Ag bonding in an ASW. The shortest Ag-O distance of $2.48 \AA$ is significantly longer than the $\mathrm{Ag}-\mathrm{O}$ bond length of $2.04 \AA$ in $\mathrm{Ag}_{2} \mathrm{O}$ (Supplementary Fig. 6 and Table 3), indicative of a weak interaction between $\mathrm{ASW}$ and $\mathrm{a}-\mathrm{MnO}_{2}$.

The geometric structures of ASWs were directly imaged by high-resolution TEM (HRTEM). Figure 2a-c shows an HRTEM image, a simulated image ${ }^{13,14}$, and a calculated diffraction pattern of ASWs viewed along an $\alpha-\mathrm{MnO}_{2}[120]$ axis, respectively. In Fig. 2a, ASWs along the $\alpha-\mathrm{MnO}_{2}[001]$ direction with the $\mathrm{Ag}-\mathrm{Ag}$ bond length of $\sim 2.87 \AA$ are clearly visible, approaching the typical $\mathrm{Ag}-\mathrm{Ag}$ bond length in bulk Ag. Similarly, parallel wires of bright atomic columns are observed along the $\alpha-\mathrm{MnO}_{2}[111]$ direction in the high angle annular dark field scanning transmission electron microscopy (HAADF-STEM) image (Fig. 2d), particularly shown in the images after Fourier filtering (Fig. 2d inset and Fig. 2e) ${ }^{14}$. As the scattering cross section in HAADF-STEM image is approximately proportional to $\mathrm{Z}^{1.8}$ (where $\mathrm{Z}$ is the atomic number $)^{15}, \mathrm{Ag}$ atoms appear brighter than $\mathrm{Mn}$ and $\mathrm{O}$ atoms. The bright linear atomic columns in the HAADF-STEM image thus 

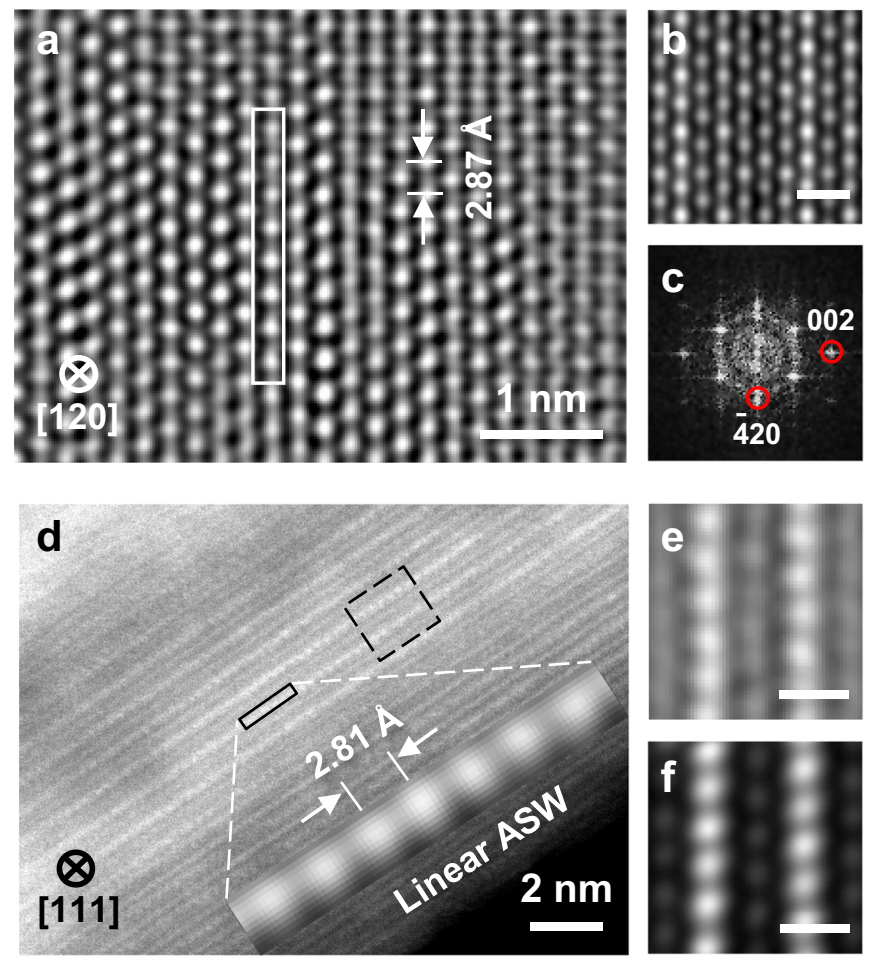
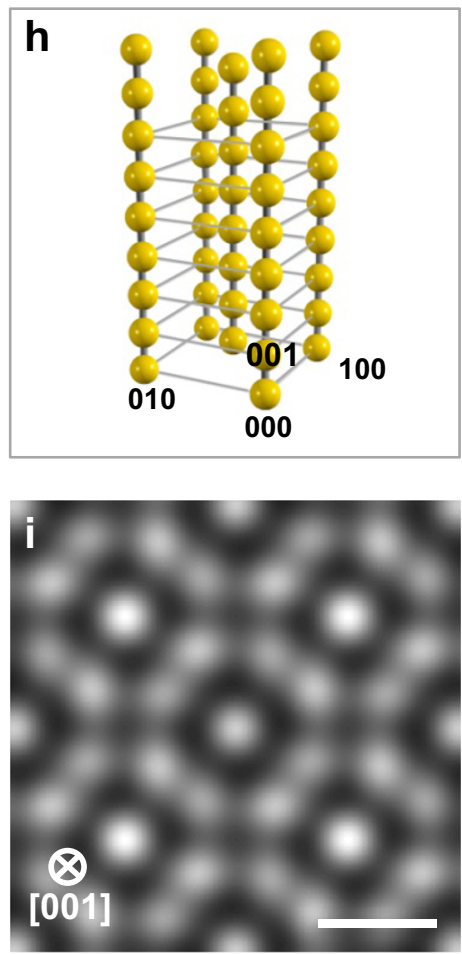

Fig. 2 Electron microscopy imaging and structure models of ASWs. a, b Filtered and simulated $\mathrm{HRTEM}$ images viewed from a $\alpha-\mathrm{MnO}_{2}[12 \mathrm{O}]$ axis. c Calculated diffraction pattern from a. d HAADF-STEM image, viewed from the $\alpha-\mathrm{MnO}_{2}[111]$ direction (the inset shows an enlarged linear Ag wire after

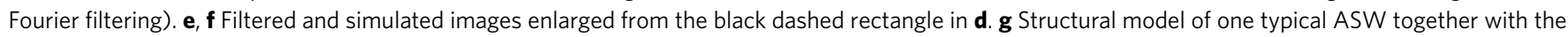
$\alpha-\mathrm{MnO}_{2}$ framework. $\mathbf{h}$ 3D model of the array with five ASWs. i Simulated image of ASWs in the $\alpha-\mathrm{MnO}_{2}$ tunnels viewed from the [001] direction showing the inter-wire distance. Scale bars in $\mathbf{b}, \mathbf{e}, \mathbf{f}$, and $\mathbf{i}, 0.5 \mathrm{~nm}$.

are ASWs, as shown by a corresponding simulated image (Fig. 2f). An observed distance of $\sim 2.81 \AA$ for a typical ASW is attributed to the projected distance of the $\mathrm{Ag}-\mathrm{Ag}$ bond length of $\sim 2.87 \AA$ on the $\alpha-\mathrm{MnO}_{2}(111)$ plane. These observations are in good accordance with the results of SXRD pattern and EXAFS spectra.

A structural model of the linear atomic wires was constructed on the basis of the above results (Fig. 2g). According to the experimental design, we filled all tunnels of the $\alpha-\mathrm{MnO}_{2}$ nanorods with $\mathrm{Ag}$ atoms to form the linear atomic $\mathrm{Ag}$ wires (Supplementary Fig. 2). As a result, the average length of ASWs is estimated to be $\sim 0.5 \mu \mathrm{m}$ with $\sim 4 \%$ of ASWs longer than $1 \mu \mathrm{m}$ (Supplementary Fig. 2). The average width of the $\mathrm{a}-\mathrm{MnO}_{2}$ nanorods is $\sim 12 \mathrm{~nm}$, and more than 280 parallel ASWs were assembled in the tunnels of each $\alpha-\mathrm{MnO}_{2}$ nanorod to form an atomic wire array (Fig. 2h). The closest-neighbor inter-wire spacing of this array is $6.95 \AA$ (Fig. 2i). Hence, the suitable 3D sizes of this array enable ASWs as one electrical device to be connected into a circuit.

Assembly process of the atomic wire array. Next we present the assembly process of ASWs. We firstly prepared $\mathrm{a}-\mathrm{MnO}_{2}$ nanorods of up to $1 \mu \mathrm{m}$ in length and over $10 \mathrm{~nm}$ in width, which have square 1D parallel tunnels extending along the [001] growth direction ${ }^{16}$. $\mathrm{Ag} / \mathrm{MnO}_{2}$ was then synthesized by depositing truncated octahedral $\mathrm{Ag} \mathrm{NPs}$ onto the $\mathrm{a}-\mathrm{MnO}_{2}$ surfaces (inset of Fig. 3a, Supplementary Fig. 7). Normally, supported metal NPs are prone to sintering and forming larger particles through thermal activation ${ }^{17,18}$ via Ostwald ripening and/or coalescence $^{19}$, depending on the particle size ${ }^{20}$. In our work, however, shrinking of $\mathrm{Ag} \mathrm{NPs}$ on the $\alpha-\mathrm{MnO}_{2}$ surfaces was clearly observed by using temperature-programmed SXRD technique, in situ TEM, and EXAFS spectroscopy.
Figure 3a shows the temperature-programmed SXRD patterns of $\mathrm{Ag} / \mathrm{MnO}_{2}$. The intensity of the SXRD pattern of Ag NPs becomes weaker and ultimately disappears as the temperature increases, indicating that Ag NPs gradually shrink and finally reach a highly dispersive state. In particular, the $\operatorname{Ag}(111)$ reflection fades as the annealing temperature increases. At $383 \mathrm{~K}, \mathrm{Ag}$ atoms become sufficiently active, detaching from Ag $\mathrm{NPs}$ and diffusing along the $\alpha-\mathrm{MnO}_{2}$ surfaces (Supplementary Fig. 8), similar to what has been observed for Ag atoms diffusing across Pt-Ag step boundaries ${ }^{6}$. As the temperature further increases to $\sim 493 \mathrm{~K}$, a clear anomaly in the first derivative SXRD patterns is observed (Supplementary Fig. 8), implying that the Ag atoms start to be assembled into the $\alpha-\mathrm{MnO}_{2}$ tunnels.

Recent work shows that the reactive environment plays a key role in the redispersion of noble metal NPs ${ }^{21}$. In order to understand the mechanism behind the novel process and rationalize the influence of atmospheres, we use an advanced in situ environmental TEM tool to record the structural evolution of $\mathrm{MnO}_{2}$ supported Ag NPs in situ and with high spatial resolutions (Supplementary Figs. 9-10). Clearly, the annealing under the inert environment cannot trigger the re-dispersion of Ag NPs, but causes the serious aggregation. In contrast, the redispersion of Ag NPs took place during the oxygen annealing, leading to the formation of ASWs. This result keeps a good agreement with the temperature-programmed SXRD data. The in situ environmental TEM results evidence that the interaction between $\mathrm{Ag}$ NPs and $\mathrm{O}_{2}$ is the driving force to enhance the mobility of $\mathrm{Ag}$ atoms ${ }^{22}$, which move from the NPs to the support and are finally hosted in the matrix ${ }^{23-25}$. As showed in Supplementary Fig. 11 and corresponding Supplementary Movie, a Ag NP collapsed and quickly disappeared in $5 \mathrm{~min}$ at $270{ }^{\circ} \mathrm{C}$ in the presence of $\mathrm{O}_{2}$. During the redispersion process, a stronger adherence of $\mathrm{Ag} \mathrm{NP}$ to $\mathrm{a}-\mathrm{MnO}_{2}$ was observed, leading to the 

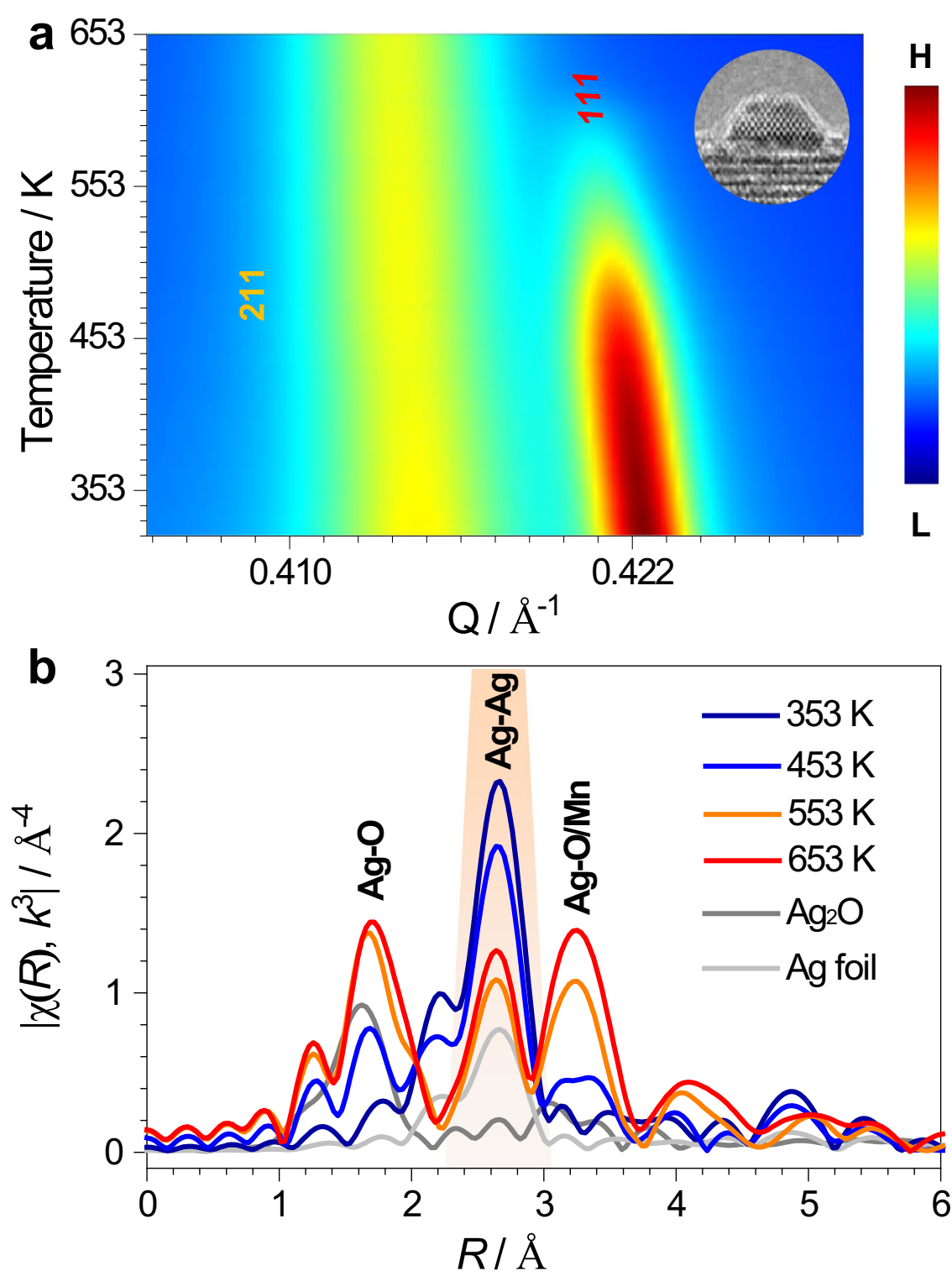

Fig. 3 Assembly process of ASWs. a Contour map of temperature-programmed SXRD patterns of $\mathrm{Ag} / \mathrm{MnO}_{2}$ as a function of momentum transfer ( $\mathrm{Q}$ ) from 323 to $653 \mathrm{~K}$, showing the diffraction intensities of the $\mathrm{Ag}(111)$ and the $\alpha-\mathrm{MnO}_{2}$ (211) reflections. Inset: $\mathrm{HRTEM}$ image of $\mathrm{Ag} / \mathrm{MnO} 2$ with a $\mathrm{Ag} \mathrm{NP}$ of 2 $\mathrm{nm}$ in height and $4 \mathrm{~nm}$ in width. $\mathrm{H}$ and $\mathrm{L}$ in the scale bar refer to High and Low in intensity, respectively. b Room-temperature ex situ Ag $K$-edge $\chi(\mathrm{R}) k^{3}$ weighted FT EXAFS spectra of $\mathrm{Ag} / \mathrm{MnO}_{2}$ after being annealed at higher temperatures, and two references of $\mathrm{Ag}_{2} \mathrm{O}$ and $\mathrm{Ag}$ foil. The amplitudes of $\mathrm{Ag} 2 \mathrm{O}$, $\mathrm{Ag}$ foil, and $\mathrm{Ag} / \mathrm{MnO}_{2}$ spectra after being annealed at $353 \mathrm{~K}$ and $453 \mathrm{~K}$ were multiplied by $1 / 4,1 / 18,1 / 4$, and $2 / 3$, respectively.

collapse of the Ag NP. It looks like that the process is surfacemediated, in which atomic species after being emitted from a metal NP diffuse on the surface of the support until being trapped by a strong metal-support interaction ${ }^{23-25}$ (Supplementary discussion).

The local structures of the Ag atoms during the thermal diffusion process were determined by using in situ EXAFS spectroscopy (Supplementary Fig. 12). To eliminate the effects of non-symmetric and inhomogeneous distribution of the instantaneous bond length owing to the thermal disorder ${ }^{26}$, the corresponding ex situ EXAFS spectra were measured after the samples were annealed at higher temperatures and then cooled down to room temperature (Fig. 3b). As the annealing temperature increases, the Fourier transform (FT) amplitude due to the first $\mathrm{Ag}-\mathrm{Ag}$ shell decreases, while the FT amplitudes due to two Ag-O shells increase. Specifically, after annealing at $653 \mathrm{~K}$, the $\mathrm{Ag}$ atoms have been assembled into the tunnels to form ASW arrays. Although the geometric size of the $\alpha-\mathrm{MnO}_{2}$ tunnel is $4.7 \AA \times 4.7 \AA$, the inner effective diameter is so small that it only allows single Ag atoms to diffuse along the tunnel at one time ${ }^{27,28}$. Thus, the atomic wire arrays are formed via the atomby-atom assembly process.

Electronic and electrical properties of the atomic wire array. Finally, we explored the electronic structures and conducting properties of ASWs. Figure 4a shows the X-ray absorption near edge structure (XANES) spectra of ASWs, metallic Ag powder and $\mathrm{Ag}_{2} \mathrm{SO}_{4}$ at the $\mathrm{Ag} L_{3}$ edge. Metallic $\mathrm{Ag}$ powder and $\mathrm{Ag}_{2} \mathrm{SO}_{4}$, respectively with the $\mathrm{Ag}^{0}\left(4 d^{10} 5 s^{1}\right)$ and $\mathrm{Ag}^{+}\left(4 d^{10} 5 s^{0}\right)$ electronic configuration are chosen as references in order to more precisely determine the electronic structures of ASWs ${ }^{15}$. No distinct peak at $\sim 3354 \mathrm{eV}$ appears in the XANES spectrum of metallic Ag because of the absence of the unoccupied $4 d$ orbitals $\left(4 d^{10}\right)$. A 

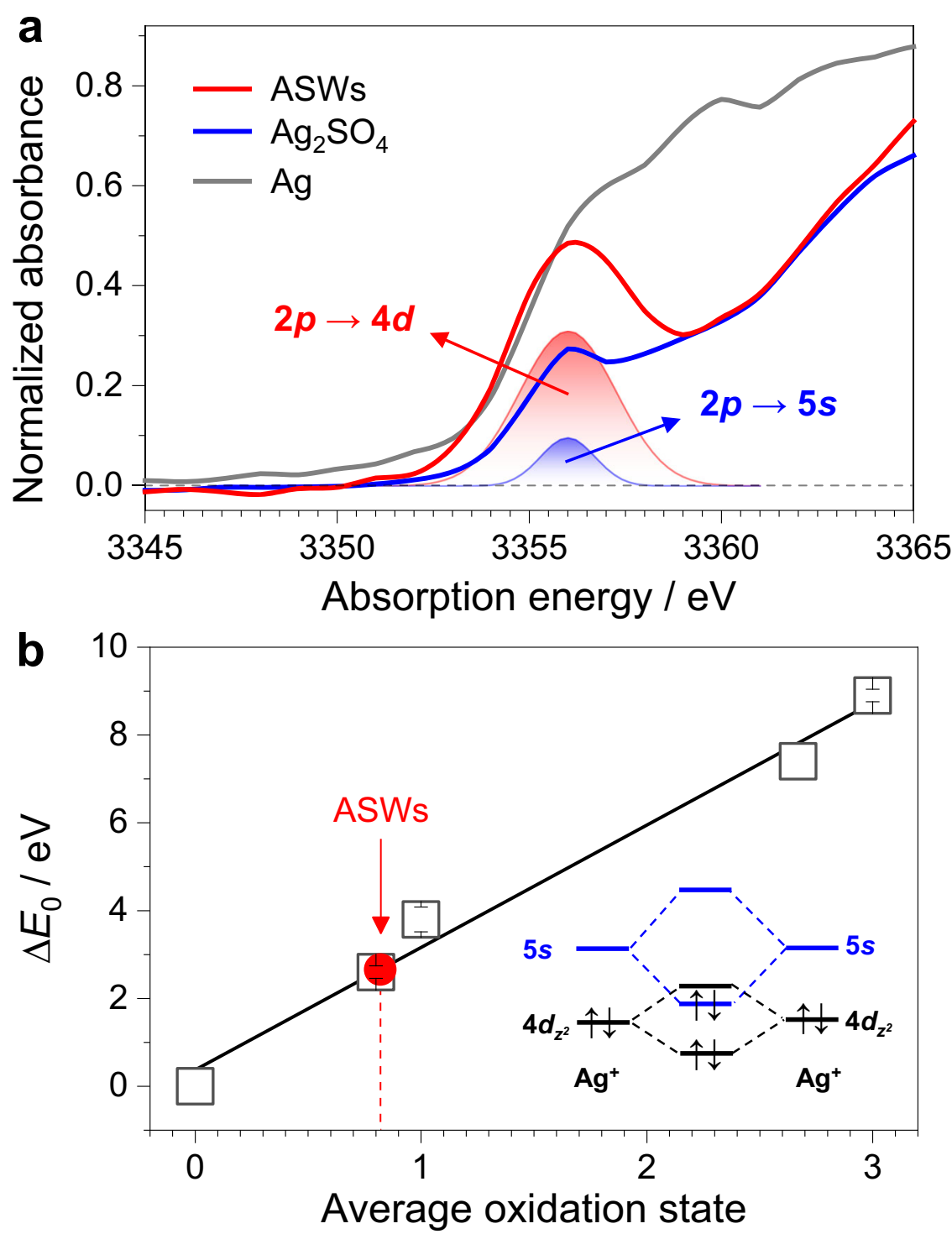

Fig. 4 Electronic structures of ASWs. a XANES spectra of ASWs and two references at the $\mathrm{Ag} \mathrm{L}_{3}$-edge. Red and blue shades represent the edge peaks of ASWs and $\mathrm{Ag}_{2} \mathrm{SO}_{4}$, respectively. $\mathbf{b}$ The change of $\mathrm{Ag} L_{1}$-edge energy $\left(\Delta E_{0}\right)$ versus average oxidation state. The Ag metal edge energy is selected as the reference energy, i.e., $\Delta E_{0}=0 \mathrm{eV}$. Data of binary silver oxides containing $\mathrm{Ag}$ in formal oxidation states of $+0.8,+1,+2.67$, and +3 were obtained from reference ${ }^{31}$. Inset: Schematic illustration of the $4 d-5$ s hybridization or overlapping among Ag atoms of ASWs leading to the $5 \mathrm{~s}$ bonding orbitals shifting down below the $4 d$ anti-bonding orbitals ${ }^{15}$.

weak peak at $3354.8 \mathrm{eV}$ was observed in the spectrum of $\mathrm{Ag}_{2} \mathrm{SO}_{4}$, which should be assigned to the $2 p_{3 / 2} \rightarrow 5 s$ transition and enhanced by the $4 d-5 s$ hybridization according to the fitting analysis (Fig. $4 \mathrm{a})^{15}$. At the same absorption energy of $3354.8 \mathrm{eV}$, a strong peak appearing in the XANES spectrum of ASWs was mainly attributed to dipole-allowed $2 p_{3 / 2} \rightarrow 4 d$ transitions, suggesting the presence of the unoccupied Ag $4 d$ orbitals $^{15}$. The unoccupied orbitals are characteristics of anti-bonding orbitals, thus reinforcing $\mathrm{Ag}-\mathrm{Ag}$ bonds ${ }^{29,30}$. Therefore, the reinforced $\mathrm{Ag}-\mathrm{Ag}$ bonds together with the scaffolding function of $\alpha-\mathrm{MnO}_{2}$ via the weak $\mathrm{Ag}-\mathrm{O}$ interactions enable ASWs to be very stable in air.

The electronic states of ASWs were further studied by using the XANES spectroscopy at the $\mathrm{Ag} L_{1}$ edge (Supplementary Fig. 13), which can precisely determine the oxidation states ${ }^{31}$. Figure $4 \mathrm{~b}$ shows the differential edge energies $\left(\Delta E_{0}\right)$ with respect to that of metallic $\mathrm{Ag}$ as a function of average oxidation states. Thus, the average oxidation state of ASWs was determined to be close to +1 (more precisely +0.82 ) according to the calibrated curve. On the basis of the results in Fig. 4a, the strong intensity of the edge peak reflects the hole in the $4 d$ orbitals. By combining the results of XANES spectra at the $\operatorname{Ag} L_{3}$ and $L_{1}$ edges, we deduced

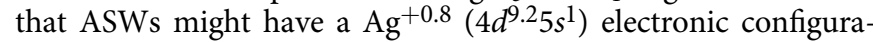
tion. This evidence implies the presence of the $4 d-5 s$ hybridization, allowing the energy of the $5 s$ bonding orbitals to be lower than that of the $4 d$ anti-bonding orbitals. It is also not difficult to interpret this configuration by using a molecular orbital theory ${ }^{15,31}$. As calculated by Behrens ${ }^{31}$, if the filled $\mathrm{Ag} 4 d_{z^{2}}$ and empty $5 s$ orbitals have the same symmetry and are hybridized, the $4 d$ vacancy concomitant with the $5 s$ occupied orbital is created (inset of Fig. 4b). As for ASWs, we set the wire direction as the $Z$ axis, and thus $4 d_{z^{2}}$ and $5 s$ orbitals have the same symmetry, resulting in the $4 d_{z^{2}}$ vacancy. In principle, the $4 d$ and $5 s$ electrons are often characteristics of localized and delocalized features, respectively ${ }^{32}$. As a consequence, the depletion of the occupied states of the $4 d_{z^{2}}$ anti-bonding orbitals strengthens the $\mathrm{Ag}-\mathrm{Ag}$ 


\section{a}
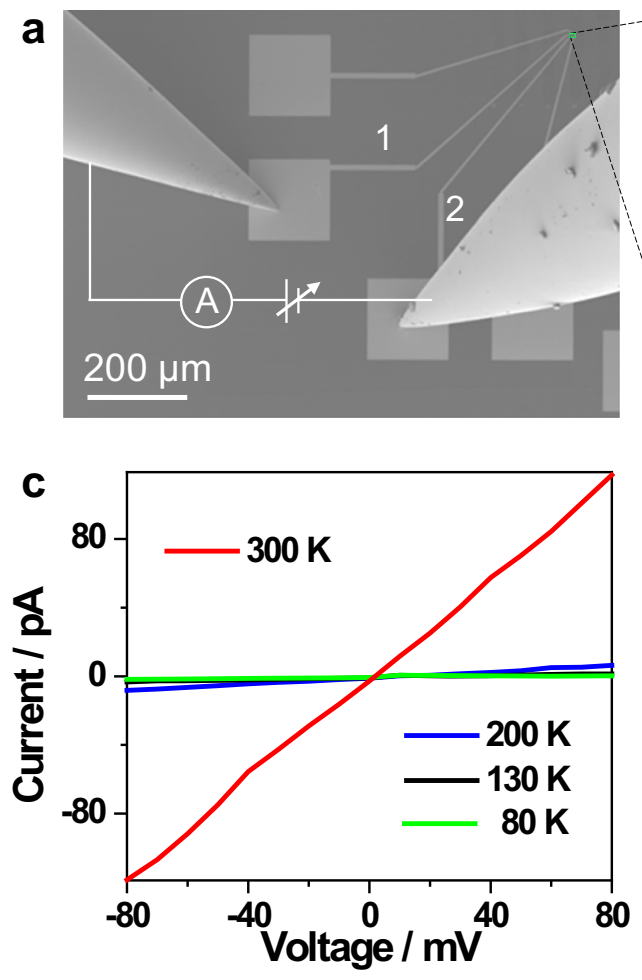
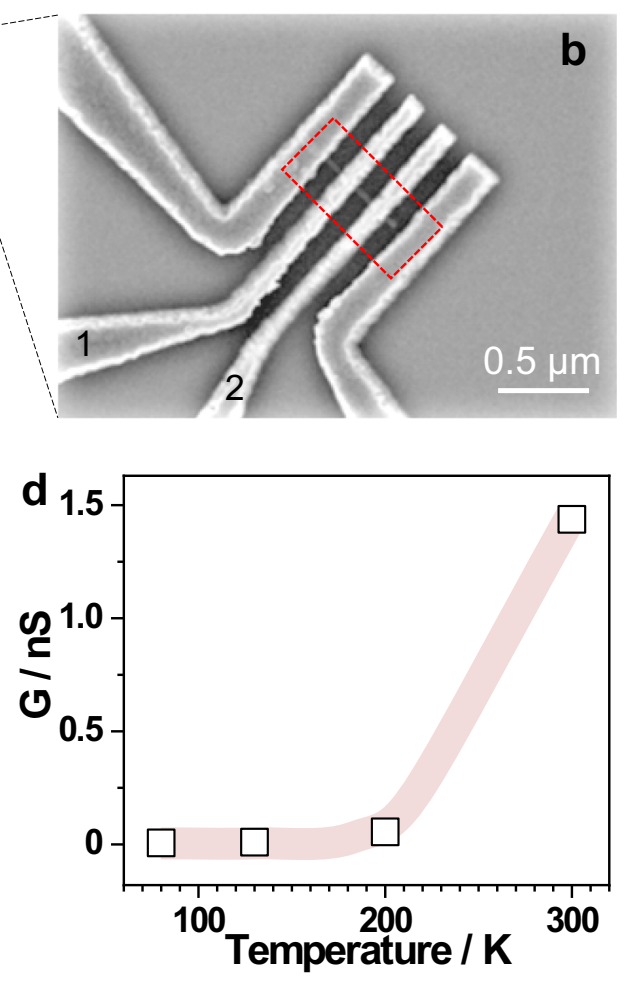

Fig. 5 Electrical properties of ASWs. a Circuit setup for the I-V measurements of ASWs. b An ASW array bridged between the nanoelectrodes (labeled as

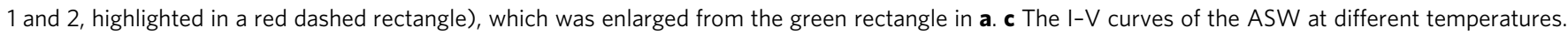
d The corresponding conductance $(\mathrm{G})$ of the array calculated from the slopes of the I-V curves (c).

bonds along the wire direction, greatly enhancing the stability of ASWs, and owing to the orbital spherical symmetry, the presence of the $5 s$ electrons with the delocalized feature endows ASWs with the conducting property.

We measured the I-V curves of metallic ASWs in a temperature range of $80-300 \mathrm{~K}$ by bridging one typical atomic wire array between two nanoelectrodes to make an electrical circuit device (Fig. 5a, b). Figure 5c,d show the I-V curves of ASWs at different temperatures and the corresponding conductance as a function of temperature, respectively. ASWs exhibit thermally sensitive conductive properties of a temperaturedependent insulator-to-metal transition. Below $200 \mathrm{~K}$, the current through ASWs is close to zero in the applied voltage range, and thus the conductance of ASWs approaches zero (Fig. 5d), implying an insulating behavior, i.e., the device is switched off. At $300 \mathrm{~K}$, the linear I-V relation of ASWs demonstrates the Ohmic conductance (Fig. 5c) with a value of $1.5 \mathrm{nS}$ (Fig. 5d) calculated from the slope of the red I-V curve in Fig. $5 c$, and, thus, the device is switched on. The I-V curve measured at $300 \mathrm{~K}$ confirms that electrons can be transferred along ASWs. Therefore, owing to the insulator-to-metal transition feature, ASWs can switch ON and OFF a circuit device, and this electrical feature allows the atomic wire array to be used as new thermal electrical devices.

\section{Discussion}

To understand possible mechanisms behind the atomic assembling, we carried out the $\mathrm{O} 1 s, \mathrm{Mn} 2 p \mathrm{X}$-ray photoelectron spectra (XPS) of the atomic wire array. Supplementary Figure 14 depicts $\mathrm{O} 1 s \mathrm{XPS}$ of $\mathrm{Ag} / \mathrm{MnO}_{2}$ and ASWs in the $\mathrm{a}-\mathrm{MnO}_{2}$ tunnels together with their difference, and only a little discrepancy in their $\mathrm{O}$ $1 s$ XPS is discernible. Subtly, after Ag NPs were transferred into ASWs in the $a-\mathrm{MnO}_{2}$ tunnels, the intensity of the O $1 \mathrm{~s}$ XPS of $\mathrm{MnO}_{2}$ slightly decreases in $532-534 \mathrm{eV}$, and increases at $530.5 \mathrm{eV}$, suggesting that the electron density of the $\mathrm{O}$ atoms slightly increases after the formation of the atomic wire array in the $\alpha$ $\mathrm{MnO}_{2}$ tunnels. The oxidation states of $\mathrm{Mn}$ are preserved after the formation of ASWs (Supplementary Fig. 15). This indicates that the interaction mainly occurs between $\mathrm{O}$ atoms of $\mathrm{a}-\mathrm{MnO}_{2}$ and $\mathrm{Ag}$ of ASWs with a small amount of the Ag $4 d$ electrons transferring to the $\mathrm{O}$ atoms of $\mathrm{a}-\mathrm{MnO}_{2}$, resulting in the partially unoccupied $\mathrm{Ag} 4 d$ states, consistent with the results obtained from the XANES spectra above. The partial depletion of the electronic states is found at the top of the $4 d$ orbitals with antibonding character ${ }^{33}$, implying an increased strength of the $\mathrm{Ag}-\mathrm{Ag}$ bonds ${ }^{34}$. As a result, $\alpha-\mathrm{MnO}_{2}$ has three main functions: (i) as a scaffold to protect ASWs from coalescence, (ii) as a special electron acceptor to only deplete the occupied states of Ag $4 d$ anti-orbitals to strengthen the Ag-Ag bonds and keep the Ag $5 s$ electrons with the delocalized states, and (iii) as an ideal container to assemble ASWs into the coherently oriented array with the suitable $3 \mathrm{D}$ sizes, which enables the array to be connected into thermal electrical devices. In fact, $\alpha-\mathrm{MnO}_{2}$ is not unique in the family of the porous materials, and we anticipate that other metal atomic wires or arrays can also be created by choosing suitable porous materials and optimizing host-guest interactions.

\section{Methods}

Sample synthesis. All the chemicals are of analytical grade and used as received.

Synthesis of ASWs. a- $\mathrm{MnO}_{2}$ was prepared by a hydrothermal route with an aqueous solution of $\mathrm{MnSO}_{4}\left(0.20 \mathrm{~mol} \mathrm{~L}^{-1}\right),\left(\mathrm{NH}_{4}\right)_{2} \mathrm{~S}_{2} \mathrm{O}_{8}\left(0.20 \mathrm{~mol} \mathrm{~L}^{-1}\right)$, and $\left(\mathrm{NH}_{4}\right)_{2} \mathrm{SO}_{4}\left(1.00 \mathrm{~mol} \mathrm{~L}^{-1}\right)$ at $393 \mathrm{~K}$ for $12 \mathrm{~h}$. The obtained sample was filtered, washed with deionized water, dried at $383 \mathrm{~K}$ for $24 \mathrm{~h}$, and calcined at $673 \mathrm{~K}$ for $4 \mathrm{~h}$. The sample is expressed as $\mathrm{A}_{x} \mathrm{Mn}_{8} \mathrm{O}_{16}(x \leq 2)^{28}$, where A denotes the tunnel sites of $a-\mathrm{MnO}_{2} \cdot \mathrm{AgNO}_{3}(0.733 \mathrm{~g})$ was dissolved in de-ionized water to get a solution (60 $\mathrm{mL})$ at room temperature, to which an aqueous ammonia solution (25 wt.\%) was slowly added under stirring until the solution became transparent. Then, both the transparent solution and an $\mathrm{H}_{2} \mathrm{O}_{2}$ solution $(30 \mathrm{wt} . \%, 30 \mathrm{~mL})$ were simultaneously added to another suspension $(80 \mathrm{~mL})$ containing $\alpha-\mathrm{MnO}_{2}(1.760 \mathrm{~g})$ under stirring 
at $298 \mathrm{~K}$ for $1 \mathrm{~h}$. The final suspension was filtered, washed with deionized water, and then dried in $353 \mathrm{~K}$ for $24 \mathrm{~h}$ to get $\mathrm{Ag}$ NPs supported on $\alpha-\mathrm{MnO}_{2}$ surfaces. Ag/ $\mathrm{MnO}_{2}$ was annealed at $653 \mathrm{~K}$ in air for $4 \mathrm{~h}$ to obtain ASWs inside $\alpha-\mathrm{MnO}_{2}$ tunnels.

Transmission electron microscopy (TEM) images. TEM, high resolution TEM (HRTEM) images, and energy-dispersive X-ray (EDX) microanalyses (point/linescan analyses) were carried out with a JEOL JEM-2100F field-emission gun transmission electron microscope operated at an accelerating voltage of $200 \mathrm{kV}$ and equipped with an ultra-high resolution pole-piece that provides a point-resolution better than $0.19 \mathrm{~nm}$. It was also equipped with a STEM control unit (Gatan), EDX detector (SDD $80 \mathrm{~mm}^{2}$ ), CCD camera (14-bit Gatan Orius SC600), bright-field (BF), and HAADF detectors (JEOL). Some microscope parameters used were as follows: defocus: $-43.4 \mathrm{~nm}$, Cs (spherical aberration): $0.5 \mathrm{~mm}, \mathrm{Cc}$ (chromatic aberration): $1.0 \mathrm{~mm}$. Fine powders of the materials were dispersed in ethanol, sonicated, and sprayed on a carbon coated copper grid, and then allowed to air-dry. Gatan SOLARUS 950 was used before observation. TEM simulations were conducted using the QSTEM v2.22 software based on multi-slice algorithm. A plane wave incident on the sample is modified by the projected potential of an atomic model partitioned into different slices. The modified complex wave is propagated through each slice of the sample.

In situ TEM image. We used an in situ gas holder (Climate S3, DENSsolutions) and a dedicated field-emission S/TEM (FEI Talos F20X) with an accelerating voltage of $200 \mathrm{kV}$ to conduct the in-situ annealing experiments under different gaseous environments. The as-prepared $\mathrm{Ag} \mathrm{NPs}$ supported on $\alpha-\mathrm{MnO}_{2}\left(\mathrm{Ag} / \mathrm{MnO}_{2}\right)$ was used as a precursor. The sample was directly dispersed in a MEMS chip for the in situ TEM experiment. After the holder equipped with the chip was inserted into TEM, different gases, $\mathrm{N}_{2}$ or $\mathrm{O}_{2}$, was introduced into the holder and the inner pressure was kept at $1 \mathrm{~atm}$ pressure. The temperature was gradually increased from room temperature to desired temperatures. Once the temperature reached at setting points, HAADF-STEM image of the sample was recorded. In order to reduce beam damage, electron beam was completely closed during the annealing treatment and the dose rate was minimized. Clearly, the imaging conditions allow for no damages to both $\mathrm{Ag} \mathrm{NPs}$ or $\alpha-\mathrm{MnO}_{2}$. We also recorded the dynamic redispersion process of the supported $\mathrm{Ag}$ nanoparticles at $270{ }^{\circ} \mathrm{C}$ under atmospheric oxygen to visualize the deconstruction of the selected nanoparticles.

Synchrotron X-ray diffraction (SXRD) patterns. The room temperature SXRD patterns were recorded at BL14B of the Shanghai Synchrotron Radiation Facility (SSRF) at a wavelength of $0.6883 \AA$. A Mar345 image plate detector was employed for the data collection and the data were further integrated using the fit $2 \mathrm{~d} \mathrm{code}^{35}$. The beam was monochromatized using a $\mathrm{Si}(111)$ crystal and a $\mathrm{Rh} / \mathrm{Si}$ mirror was used for the beam focusing to a size of $\sim 0.5 \times 0.5 \mathrm{~mm}^{2}$. Rietveld refinements of the diffraction data were performed with the FULLPROF software package on the basis of the space group $I 4 / \mathrm{m}$. The temperature-programmed SXRD patterns were collected at BL14B of SSRF at a wavelength of $1.2398 \AA$. A typical amount of the sample $(\sim 1.5 \mathrm{mg})$ was loaded in a quartz capillary tube with a diameter of $\sim 1 \mathrm{~mm}$, and then heating was carried out using a temperature-programmed procedure at a ramp of $3 \mathrm{~K} \mathrm{~min}^{-1}$. Each SXRD pattern was collected at 3-min intervals and analyzed by using a CMPR software.

X-ray absorption spectra. X-ray absorption spectra at the $\mathrm{Ag} K$-edge were measured at BL14W of the SSRF with an electron beam energy of $3.5 \mathrm{GeV}$ and a ring current of 200-300 mA. The data were collected with a fixed exit monochromator using two flat $\mathrm{Si}(311)$ crystals. Harmonics were rejected by using a grazing incidence mirror. The XANES spectra at the $\mathrm{Ag} L_{1,3}$-edge were acquired at BL4B7A of the Beijing Synchrotron Radiation Facility (BSRF) with an electron beam energy of $2.2 \mathrm{GeV}$ and a ring current of $300-450 \mathrm{~mA}$. The energy step for XANES measurement was set to be $0.2 \mathrm{eV}$. The extended X-ray absorption fine structure (EXAFS) spectra were collected in a transmission mode using ion chambers filled with $\mathrm{N}_{2}$. The raw data were analyzed by using the IFEFFIT 1.2.11 software package.

X-ray photoelectron spectra (XPS). XPS were recorded using Kratos Axis UltraDLD system with a charge neutralizer and a $150 \mathrm{~W}$ Al (Mono) X-ray gun (1486.6 $\mathrm{eV}$ ) equipped with a delay-line detector (DLD). The spectra were acquired at a normal emission with a passing energy of $40 \mathrm{eV}$. The spectra were calibrated according to the $\mathrm{C} 1 \mathrm{~s}$ peak at $284.6 \mathrm{eV}$.

\section{Electrical measurements by in situ scanning electronic microscopy (SEM)}

technique. Samples were dispersed in ethanol and dropped on a marked Si substrate with $300 \mathrm{~nm} \mathrm{SiO}$ layer. The position of the suitable individual wire array was determined by SEM observations (JSM-6700F). The Cr/Au (10 nm/100 nm) electrodes were patterned onto the top of the atomic wire array using lithography and electron-beam deposition followed by a lift-off process. Then, the wafer was transferred into a SEM with piezoelectric micromanipulators (Kleindiek). Finally, temperature-dependent electrical measurements were carried out by using a semiconductor characterization system (Keithley 4200) and a home-made cooling stage.

\section{Data availability}

All the data that support the findings of this study are available from the corresponding author upon reasonable request. Source data are provided with this paper.

Received: 16 July 2020; Accepted: 27 January 2021;

Published online: 19 February 2021

\section{References}

1. Yanson, A. I., Bollinger, G. R., van den Brom, H. E., Agrait, N. \& van Ruitenbeek, J. M. Formation and manipulation of a metallic wire of single gold atoms. Nature 395, 783-785 (1998)

2. Ohnishi, H., Kondo, Y. \& Takayanagi, K. Quantized conductance through individual rows of suspended gold atoms. Nature 395, 780-783 (1998).

3. Nilius, N., Wallis, T. M. \& Ho, W. Development of one-dimensional band structure in artificial gold chains. Science 297, 1853-1856 (2002).

4. Crain, J. N. \& Pierce, D. T. End states in one-dimensional atom chains. Science 307, 703-706 (2005).

5. Chen, C., Bobisch, C. A. \& Ho, W. Visualization of Fermi's golden rule through imaging of light emission from atomic silver chains. Science 325, 981-985 (2009).

6. Gambardella, P., Blanc, M., Brune, H., Kuhnke, K. \& Kern, K. Onedimensional metal chains on Pt vicinal surfaces. Phys. Rev. B. 61, 2254-2262 (2000).

7. Höhn, P. et al. $\left(\mathrm{Ca}_{7} \mathrm{~N}_{4}\right)\left[\mathrm{M}_{\mathrm{x}}\right](\mathrm{M}=\mathrm{Ag}, \mathrm{Ga}, \mathrm{In}, \mathrm{Tl})$ : Linear metal chains as guests in a subnitride host. Angew. Chem. Int. Ed. 45, 6681-6685 (2006).

8. Hirjibehedin, C. F., Lutz, C. P. \& Heinrich, A. J. Spin coupling in engineered atomic structures. Science 312, 1021-1024 (2006).

9. Rodrigues, V., Fuhrer, T. \& Ugarte, D. Signature of atomic structure in the quantum conductance of gold nanowires. Phys. Rev. Lett. 85, 4124-4127 (2000).

10. Rietveld, H. M. A profile refinement method for nuclear and magnetic structures. J. Appl. Cryst. 2, 65-71 (1969).

11. Ling, C. \& Mizuno, F. Capture lithium in $\alpha \mathrm{MnO}_{2}$ : Insights from first principles. Chem. Mater. 24, 3943-3951 (2012).

12. Moon, H. R., Choi, C. H. \& Suh, M. P. A stair-shaped molecular silver(0) chain. Angew. Chem. Int. Ed. 47, 8390-8393 (2008).

13. Koch, C. Determination of Core Structure Periodicity and Point Defect Density Along Dislocations. PhD thesis. Arizona State University (2002).

14. Koch, C., Spence, J. C. H., Zorman, C., Mehregany, M. \& Chung, J. Modelling of HREM and nanodiffraction for dislocation kinks and core reconstruction. $J$. Phys. Condens. Mat. 12, 10175-10183 (2000).

15. Miyamoto, T., Niimi, H., Kitajima, Y., Naito, T. \& Asakura, K. Ag $L_{3}$-edge Xray absorption near-edge structure of $4 \mathrm{~d}_{10}\left(\mathrm{Ag}^{+}\right)$compounds: Origin of the edge peak and its chemical relevance. J. Phys. Chem. A 114, 4093-4098 (2010).

16. Huang, Z. et al. Catalytically active single-atom sites fabricated from silver particles. Angew. Chem. Int. Ed. 51, 4198-4203 (2012)

17. Farmer, J. A. \& Campbell, C. T. Ceria maintains smaller metal catalyst particles by strong metal-support bonding. Science 329, 933-936 (2010).

18. Lei, Y. et al. Increased silver activity for direct propylene epoxidation via subnanometer size effects. Science 328, 224-228 (2010).

19. Bowker, M. The going rate for catalysts. Nat. Mater. 1, 205-206 (2002).

20. Campbell, C. T., Parker, S. C. \& Starr, D. E. The effect of size-dependent nanoparticle energetics on catalyst sintering. Science 298, 811-814 (2002).

21. Van Deelen, T. W., Mejía, C. H. \& de Jong, K. P. Control of metal-support interactions in heterogeneous catalysts to enhance activity and selectivity. Nat. Catal. 2, 955-970 (2019).

22. Gänzler, A. M. et al. Tuning the structure of platinum particles on ceria in situ for enhancing the catalytic performance of exhaust gas catalysts. Angew. Chem. Int. Ed. 56, 13078-13082 (2017).

23. Yao, Y. et al. High temperature shockwave stabilized single atoms. Nat. Nanotechnol. 14, 851-857 (2019).

24. Lang, R. et al. Non defect-stabilized thermally stable single-atom catalyst. Nat Commun. 10, 234 (2019)

25. Liu, K. et al. Strong metal-support interaction promoted scalable production of thermally stable single-atom catalysts. Nat. Commun. 11, 1263 (2020).

26. Bordiga, S., Groppo, E., Agostini, G., van Bokhoven, J. A. \& Lamberti, C. Reactivity of surface species in heterogeneous catalysts probed by in situ X-ray absorption techniques. Chem. Rev. 113, 1736-1850 (2013).

27. Kijima, N., Ikeda, T., Oikawa, K., Izumi, F. \& Yoshimura, Y. Crystal structure of an open-tunnel oxide $\alpha-\mathrm{MnO}_{2}$ analyzed by Rietveld refinements and MEMbased pattern fitting. J. Solid State Chem. 177, 1258-1267 (2004).

28. Chang, F. M. \& Jansen, M. $\mathrm{Ag}_{1.8} \mathrm{Mn}_{8} \mathrm{O}_{16}$ : Square planar coordinated $\mathrm{Ag}^{\oplus}$ ions in the channels of a novel hollandite variant. Angew. Chem. Int. Ed. 23, 906-907 (1984). 
29. Thijssen, W. H. A., Strange, M., de Brugh, J. M. J. A. \& van Ruitenbeek, J. M. Formation and properties of metal-oxygen atomic chains. N. J. Phys. 10, 033005 (2008)

30. Thijssen, W. H. A. Molecule-assisted Atomic Chain Formation. $\mathrm{PhD}$ thesis, Leiden University (2007)

31. Behrens, P., A $\beta$ mann, S., Bilow, U., Linke, C. \& Jansen, M. Electronic structure of silver oxides investigated by AgL XANES spectroscopy. Z. Anorg. Allg. Chem. 625, 111-116 (1999).

32. Goh, J. \& Akola, J. Superatom model for Ag-S nanocluster with delocalized electrons. J. Phys. Chem. C. 119, 21165-21172 (2015).

33. Thijssen, W. H. A., Marjenburgh, D., Bremmer, R. H. \& van Ruitenbeek, J. M. Oxygen-enhanced atomic chain formation. Phys. Rev. Lett. 96, 026806 (2006).

34. Smit, R. H. M., Untiedt, C., Yanson, A. I. \& van Ruitenbeek, J. M. Common origin for surface reconstruction and the formation of chains of metal atoms. Phys. Rev. Lett. 87, 266102 (2001).

35. Hammersley, A. P., Svensson, S. O., Hanfland, M., Fitch, A. N. \& Hausermann, D. Two-dimensional detector software: from real detector to idealised image or two-theta scan. High. Press. Res. 14, 235-248 (1996).

\section{Acknowledgements}

We sincerely thank Prof. John Miao and Dr. Z. Amghouz for the help in the HAADFSTEM and EDX measurement, respectively. This work is supported by NSFC (21777030 and 21976037), the National Engineering Laboratory for Flue Gas Pollution Control Technology and Equipment (NEL-KF-201903), and the National Engineering Laboratory for Mobile Source Emission Control Technology (NELMS2018B02). D.T. acknowledges the support by JSPS Kakenhi (Grant JP20K05281, JP25820336) and World Premier International (WPI) Center for Materials Nanoarchitectonics (MANA) of the National Institute for Materials Science (NIMS), Tsukuba, Japan. D.G. is grateful to the Australian Research Council (ARC) for granting a Laureate Fellowship FL160100089. X.L. acknowledges the support by NSFC $(21872163,22072090,21991153,21991150)$. Both in situ SXRD patterns and Ag K-edge X-ray absorption spectra were conducted at the Shanghai Synchrotron Radiation Facility (SSRF), Shanghai, China. The Ag $L_{1,3}$-edge Xray absorption spectrum measurements were conducted at the Beijing Synchrotron Radiation Facility (BSRF), Beijing, China.

\section{Author contributions}

X.T. directed the project. Y.C. and Z.H. prepared the catalysts, conducted the experiments, and analyzed the data. D.T., J.C., T.S., Y.B. and D.G. conducted temperature-dependent electrical measurements. X.L. and X.W. conducted the in-situ TEM experiments. W.Q., J.C., D.X. and X.H. assisted with catalyst preparation and characterization. X.T., Y.C. and Z.H. wrote the manuscript. All authors commented on the manuscript.

\section{Competing interests}

The authors declare no competing interests.

\section{Additional information}

Supplementary information The online version contains supplementary material available at https://doi.org/10.1038/s41467-021-21462-3.

Correspondence and requests for materials should be addressed to X.T.

Peer review information Nature Communications thanks Yurii Gun'ko, Arijit Sen and the other, anonymous, reviewer(s) for their contribution to the peer review of this work. Peer reviewer reports are available.

Reprints and permission information is available at http://www.nature.com/reprints

Publisher's note Springer Nature remains neutral with regard to jurisdictional claims in published maps and institutional affiliations.

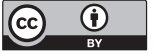

Open Access This article is licensed under a Creative Commons Attribution 4.0 International License, which permits use, sharing, adaptation, distribution and reproduction in any medium or format, as long as you give appropriate credit to the original author(s) and the source, provide a link to the Creative Commons license, and indicate if changes were made. The images or other third party material in this article are included in the article's Creative Commons license, unless indicated otherwise in a credit line to the material. If material is not included in the article's Creative Commons license and your intended use is not permitted by statutory regulation or exceeds the permitted use, you will need to obtain permission directly from the copyright holder. To view a copy of this license, visit http://creativecommons.org/ licenses/by/4.0/.

(C) The Author(s) 2021 